\title{
Inactivated Poliovirus Vaccine
}

National Cancer Institute

\section{Source}

National Cancer Institute. Inactivated Poliovirus Vaccine. NCI Thesaurus. Code C91715.

A vaccine consisting of inactivated poliovirus (IPV) types 1,2 and 3, with active immunizing activity ag ainst poliomyelitis. Upon intramuscular vaccination, inactivated poliovirus vaccine (IPV) activates the immune system to develop antibodies against polioviruses. 